\title{
Author Correction: High-resolution radar road segmentation using weakly supervised learning
}

Itai Orr ${ }^{(D,}$, Moshik Cohen and Zeev Zalevsky

Correction to: Nature Machine Intelligence https://doi.org/10.1038/s42256-020-00288-6, published online 1 February 2021.

In the version of this Article originally published, the colour bars labelled 'Power (dB)' in Figs. 1-3,5,6 were incorrect; they should have been labelled 'Amplitude (dB)'. In Fig. 5d, the colour bar labelled 'Confidence level (dB)' was incorrect; it should have been labelled 'Amplitude (dB)'. This has now been corrected.

Published online: 10 February 2021

https://doi.org/10.1038/s42256-021-00314-1

(c) The Author(s), under exclusive licence to Springer Nature Limited 2021 